\title{
EFFECT OF HYPEROSIDE ON THE INHIBITION OF ADIPOGENESIS IN 3T3-L1 ADIPOCYTES
}

\author{
M. Berkoz \\ Yuzuncu Yil University, Faculty of Pharmacy - Department of Biochemistry, Van, Turkey
}

\begin{abstract}
Context. The inhibition of adipocyte differentiation has a significant role on the prevention of obesity and obesity-associated complications.

Objective. In this study, we aimed to detect whether hyperoside is able to inhibit the conversion of pre-adiposits into mature adiposits.

Design and Methods. 3T3-L1 pre-adipocytes were stimulated so as to differentiate into mature adipocytes. Hyperoside in non-cytotoxic concentrations $(1,2,5$, and $10 \mu \mathrm{M})$ were separately applied to differentiated 3T3-L1 cells. Oil red O staining was performed and triacylglycerol contents were measured. Furthermore, gene and protein expressions of transcription factors, adipogenic genes and adipokines were examined in order to investigate the effect of hyperoside on adipocyte differentiation.

Results. Hyperoside in high concentrations significantly suppressed the adipogenic process by inhibiting the expression of transcription factors and adipogenic genes and reducing lipid accumulation in adipocytes $(\mathrm{p}<0.05)$. Low doses of hyperoside are able to inhibit adipogenesis, but higher doses are needed to reduce fat accumulation in mature adipocytes. In the case of maturing preadipocytes, $5 \mu \mathrm{M}$ of hyperoside exerts its antiadipogenic effect at the early stages of adipogenesis, whereas $10 \mu \mathrm{M}$ of hyperoside acts at the later stages $(\mathrm{p}<0.05)$.

Conclusion. These results suggest that hyperoside has a beneficial effect on the prevention and treatment of obesity.
\end{abstract}

Key words: adipogenesis, hyperoside, transcriptional factors, adipogenic genes, adipokines, 3T3L1 adipocytes.

\section{INTRODUCTION}

Obesity has become a globally prevalent health risk and is no longer considered to be only a cosmetic problem affecting certain individuals. It is associated with heart diseases, hypertension, cancer, and type 2 diabetes. In Turkey, the number of obese people is also increasing rapidly and has almost doubled since 1980
(1). In obesity, excessive fat accumulation is stored as white triglycerides in the white adipose tissue. The main function of white adipose tissue is to store large amounts of excess calories in adipocytes. Furthermore, white adipose tissue functions as an endocrine organ, secreting an array of hormones, cytokines, and fatty acids. As long as adipocytes are able to safely store and maintain excess fat, as well as exert its normal endocrine functions, there are no adverse metabolic effects (2). However, through the progression of obesity, overexpansion and chronic inflammation in adipocyte results in the elevated release of free fatty acids into circulation. As excess circulating free fatty acids continue to accumulate in non-adipose tissues, the process of lipotoxicity begins. During this process, excess spillover of fatty acids into non-adipose organs leads to cellular dysfunction, reduced glucose uptake, and impaired insulin secretion, thereby contributing to systemic insulin resistance displayed in obesity-induced diabetes mellitus. However, one way to improve sensitivity is by increasing the capacity of adipose tissue to form new and healthy adipocytes. Through the process of adipocyte differentiation, preadipocytes differentiate into mature adipocytes that are capable of storing fat (3).

Adipocyte differentiation plays a crucial role in maintaining metabolic homeostasis in the body, as this process provides adipocytes that safely store fat under tight hormonal control. During adipocyte differentiation, the shape of the cell undergoes significant changes, from fibroblast-like to spherical in shape. Adipocyte differentiation is largely divided into the following stages: early stage, intermediate stage, and terminal stage. These stages of differentiation are characterized by changes in the expression of key transcription factors, which include activating protein-1 (AP-1), the families of CCAAT-enhancerbinding proteins $\alpha$ and $\beta(C / E B P \alpha$ and $C / E B P \beta)$ and peroxisome proliferator-activated receptors (PPARs). Other transcription factors involved include signal

*Correspondence to: Mehmet Berkoz MD, "Yuzuncu Yil” University, Faculty of Pharmacy, Department of Biochemistry, Zeve Campus, Van, 65080, Turkey, E-mail: mehmet_berkoz@yahoo.com 
transducers and activators of transcription (STATs), kruppel-like factor (KLF) proteins, and sterol regulatory element-binding protein-1 (SREBP-1). At the early stage of differentiation, preadipocytes resemble a fibroblast, and during this stage, preadipocytes express early markers of differentiation, such as Pref-1 and collagen type IV. The early stage is characterized by growth, as preadipocytes proliferate to reach confluence, by which a cell-cell contact occurs. Upon reaching confluence, preadipocytes enter a resting phase referred to as growth arrest, which is required for adipocyte differentiation to proceed. At growth arrest, preadipocytes display increased expression of $C / E B P \alpha$ and $P P A R \gamma$ genes, which work in conjunction to promote differentiation $(3,4)$.

Differentiation marks the transition from the early stage to the intermediate stage. At this time, preadipocytes must receive appropriate signals for differentiation to proceed. As DNA replication and clonal amplification occur, the expression of Pre- 1 is decreased, followed by increases in the expression of $C / E B P \beta, C / E B P \alpha$, and PPAR $\gamma$ genes (4).

The differentiation process transitions from the intermediate stage to the terminal stage, which is marked by an increase in de novo lipogenesis, whereby adipocytes acquire sensitivity to insulin. This stage of differentiation is also marked by increased expression of glucose transporters and insulin receptors, as well as rate-limiting enzymes acetyl CoA carboxylase $(A C C)$ and fatty acid synthase (FASN), both of which are involved in fatty acid synthesis, and other lipogenic and lipolytic enzymes. Although the differentiation is partitioned largely into several stages, much of our understanding of the transcriptional activities that occur throughout differentiation is through the use of in vitro cell lines $(3,4)$.

Complementary and alternative medicine is an attractive therapy for obesity and obesity related diseases because it has fewer side effects, and less toxicity compared with medicinal therapy. Although herbal extracts and phytochemicals have been in folkloric medicine for hundreds of years, there are few in vivo or in vitro studies explaining the activity and mechanism of these substances (5).

Flavonoids have a number of structural characteristics that contribute to their potent activities in plants. For example, their phenolic structure gives them strong antioxidant and metal chelating properties. As conjugated aromatic compounds, they screen UV light, modulate light in the $350-450 \mathrm{~nm}$ range, and provide pigmentation. Their steroidal structure allows for control of plant growth and development, and, as polymers, they have an increased capacity to bind proteins, including enzymes, and other polymers. Flavonoids exist widely in plant-based foods and beverages. Common sources include fruits and vegetables, grains, legumes, tea, and red wine. Epidemiological studies suggest that a high flavonoid intake is associated with a reduced risk of obesity, cardiovascular disease and certain cancers. Flavonoids exert diverse physiological and biological effects in vitro and in vivo: they exhibit antiallergenic, antibacterial, anti-inflammatory, antimutagenic, and antiviral properties (6). Hyperoside (quercetin 3-O- $\beta$-dgalactoside) is a flavonol obtained from Rhododendron brachycarpum G. Don. Hyperoside shows antioxidant, antitumoural, antibacterial, antiviral, anti-inflammatory, and neuroprotective activities (7). Although the activity of quercetin anti-obesity has been studied extensively, the effect on anti-obesity of hyperoside, which has a similar molecular structure to quercetin, is not sufficiently known (5). This study aimed to investigate the effect of hyperoside on intracellular triglyceride accumulation in both preadipocytes and mature adipocytes. For this purpose, the expressions of the adipokines, factors involved in adipogenesis and triglyceride metabolism, were examined.

\section{MATERIALS AND METHODS}

\section{Reagents}

Hyperoside was obtained from Sigma-Aldrich, USA. All cell culture chemicals were bought either from Sigma-Aldrich, Merck or other standard suppliers. 3T3-L1 mouse embryo preadipocyte cell line was purchased from the Health Protection Agency (HPA) cell culture collection (Sigma 86052701). Specific primers were designed using primer3web program (v. 4.1.0) and synthesized by PRZ BioTECH (Bilkent, Ankara, Turkey).

\section{Experimental Design}

Frozen 3T3-L1 cells were immediately thawed and transferred into a $10 \mathrm{~cm}^{2}$ culture dish with culture medium (DMEM, 10\% bovine calf serum (BCS), and $1 \%$ penicillin/streptomycin). The cells were incubated in a humidified cell culture incubator with $5 \% \mathrm{CO}_{2}$ at $37^{\circ} \mathrm{C}$. The medium was replaced the following day. When the 3T3-L1 cells reached $70 \%$ confluence, cells were subcultured by adding $1 \mathrm{~mL}$ Trypsin-EDTA solution into the culture dish and incubated for 5 minutes at $37^{\circ} \mathrm{C}$. Culture medium was added to the culture dish, and then cell suspension was transferred 
into a new $10 \mathrm{~cm}^{2}$ culture dish, which included $10 \mathrm{~mL}$ of culture medium (8). To determine the number of cells that needed to be seeded for experiments, $10 \mu \mathrm{L}$ of the cell suspension was loaded onto a hemocytometer. Cells in the four squares of the hemocytometer were counted, averaged, and multiplied by 104 (9).

\section{Cell Viability Assay}

To determine the concentrations of hyperoside that did not exhibit harmful effects on cell viability ( $\leq 10 \%$ cytotoxicity), 3-[4,5-dimethylthiazole-2-yl]2,5-diphenyltetrazolium bromide (MTT) assay was performed (10).

\section{Cell Treatment}

The cells were then seeded at 50,000 cells $/ \mathrm{mL}$ in 6-well, 12-well, 96-well plates with culture medium, and this is day 0 of the experiment. The cells were incubated in the cell culture incubator. On day 2, when the cells reached $100 \%$ confluence, the culture medium was switched to differentiation media (DMEM, $10 \%$ fetal bovine serum (FBS), 1\% penicillin/streptomycin, $10 \mu \mathrm{g} /$ $\mathrm{mL}$ insulin, $0.5 \mathrm{mM}$ IBMX, $0.25 \mathrm{mM}$ dexamethasone, and $100 \mathrm{ng} / \mathrm{mL}$ biotin) including $1,2,5$, and $10 \mu \mathrm{M}$ of hyperoside (non-cytotoxic concentrations) were provided to the cells and then cells were incubated in the cell culture incubator. As negative control, only differentiation medium was applied to the cells. On day 4 , the differentiation medium was removed and replaced with post-differentiation medium (DMEM, 10\% FBS, $1 \%$ penicillin/streptomycin, and $1 \mu \mathrm{g} / \mathrm{mL}$ insulin). The post-differentiation medium was refreshed every other day until day 18 (8).
Oil Red $O$ Staining and Triacylglycerol

\section{Content of 3T3-L1 Adipocytes}

During the differentiation process, lipids are accumulated as a single lipid droplet in the cytosol of the cell. This indicates that white pre-adipocytes have developed into mature white adipocytes. To examine whether the treatment of hyperoside in the different growth stages of 3T3-L1 cells affects the differentiation process, lipid droplets in fully differentiated mature white adipocytes (day 18) were stained using Oil Red $O$. The stained oil droplets were dissolved in isopropyl alcohol, and relative triglyceride content was quantified by reading the absorbance at $490 \mathrm{~nm}$ on a microplate reader (11).

\section{Time PCR \\ RNA Preparation and Quantitative Real-}

Total RNA was extracted using TriPure RNA isolation kit (Roche Diagnostics, Mannheim, Germany) (Catalog No: 11667165001), according to the manufacturer's protocol. The total RNA $(2 \mu \mathrm{g})$ was used for cDNA synthesis using First Strand cDNA synthesis kit (Promega Corporation, Wisconsin, USA) (Catalog No: E6560S). Then mRNA expressions of PPAR $\gamma$, SREBP-1c, deacetylase sirtuin 1 (SIRT1), lipoprotein lipase $(L P L), C / E B P \alpha$ and $C / E B P \beta$, adipose triglyceride lipase $(A T G L)$, hormone sensitive lipase $(H S L), A C C, F A S N$, leptin, adiponectin, visfatin, and apelin were quantitatively determined by Roche LightCycler 480 II Real-Time PCR device using Invitrogen Universal EXPRESS SYBR GreenER qPCR SuperMixes and Two-Step qRT-PCR kit. Real-Time PCR was performed according to the methods of Lasa

Table 1. Primers for PCR amplification of each studied gene

\begin{tabular}{|c|c|c|}
\hline Gene Name & Forward Primer & Reverse Primer \\
\hline$P P A R \gamma$ & 5'-ATT CTG GCC CAC CAA CTT CGG-3' & 5'-TGG AAG CCT GAT GCT TTA TCC CCA-3' \\
\hline$C / E B P \alpha$ & 5'-TTC CTC CGG CTA AGA CTT AGC C-3' & 5'-CAG GGG TGT GTG TAT GAA CTG G-3' \\
\hline$C / E B P \beta$ & 5'-GAG CGA CGA GTA CAA GAT GCG-3' & 5'-GCT GCT CCA CCT TCT TCT GC-3' \\
\hline$S R E B P-1 c$ & 5'- GAC GCT CAT TGG CCT GG -3' & 5'- CTC TGG AGG CAG ACG ACA AG -3' \\
\hline$A T G L$ & 5'-CAC TTT AGC TCC AAG GAT GA-3' & 5'-TGG TTC AGT AGG CCA TTC CT-3' \\
\hline$H S L$ & 5'-GGT GAC ACT CGC AGA AGA CAA TA-3' & 5'-GCC GCC GTG CTG TCT CT-3' \\
\hline$L P L$ & 5'-CAG CTG GGC CTAACT TTG AG-3' & 5'-CCT CTC TGC AAT CAC ACG AA-3' \\
\hline FASN & 5'-AGC CCC TCA AGT GCA CAG TG-3' & 5'-TGC CAA TGT GTT TTC CCT GA-3' \\
\hline$A C C$ & 5'-GGA CCA CTG CAT GGA ATG TTA A-3' & 5'-TGA GTG ACT GCC GAA ACA TCT C-3' \\
\hline SIRT1 & 5'-GAC GAC GAG GGC GAG GAG-3' & 5'-ACA GGA GGT TGT CTC GGT AGC-3' \\
\hline Leptin & 5'- TGG ACC AGA CTC TGGCAG TC -3' & 5'-AGG ACA CCA TCCAGG CTC TC-3' \\
\hline Adiponectin & 5'-TG TAG GAT TGT CAGTGG ATC TG-3' & 5'-GCT CTT CAG TTGTAG TAA CGT CAT C-3' \\
\hline Visfatin & 5'-CCG GCC CGA GAT GAA TGC-3' & 5'-GGA ATA AAC TTT GCT TGT GTT GGG-3' \\
\hline Apelin & 5'-ATT TAA GGA CACGCT GAT CAA AGG-3' & 5'-AGT CCC GAA AGT ATT CAAAAG CAG-3' \\
\hline$\beta$-Actin & 5'-TCT ATG AGG GCTACG CTC TCC-3' & 5'-CAC GCT CGG TCA GGATCT TC-3' \\
\hline
\end{tabular}

(PPARY: Peroxisome proliferator-activated receptor $\gamma, C / E B P \alpha$ : CCAAT-enhancer-binding proteins alpha, C/EBP $\beta$ : CCAAT-enhancer-binding proteins beta, SREBP-1c: sterol regulatory element-binding protein-1c, ATGL: Adipose triglyceride lipase, HSL: Hormone sensitive lipase, LPL: Lipoprotein lipase, FASN: Fatty acid synthase, ACC: Acetyl-CoA carboxylase, SIRT1: deacetylase sirtuin 1). 
et al. (8) and Eseberri et al. (9). To quantify the results from real-time PCR, the threshold cycle $(\mathrm{Ct})$ values from the amplified target genes were incorporated into the $2^{-\Delta \Delta C t}$ formula. $\beta$-actin was used as the housekeeping gene. The primers used in this experiment are shown in Table $1(8,9)$.

\section{Western Blot Analysis}

Total proteins of adipocytes were obtained using Trizol (Invitrogen, Carlsbad, CA, USA), and the total protein concentration of the supernatant was measured using Pierce ${ }^{\mathrm{TM}}$ BCA Protein Assay Kit (Thermo Fisher Scientific Inc., Massachusetts, USA). Total protein was loaded into sodium dodecyl sulphatepolyacrylamide gel electrophoresis (SDS-PAGE) (Bio-Rad Laboratories, Hercules, California, USA), the protein bands were transferred to polyvinylidene

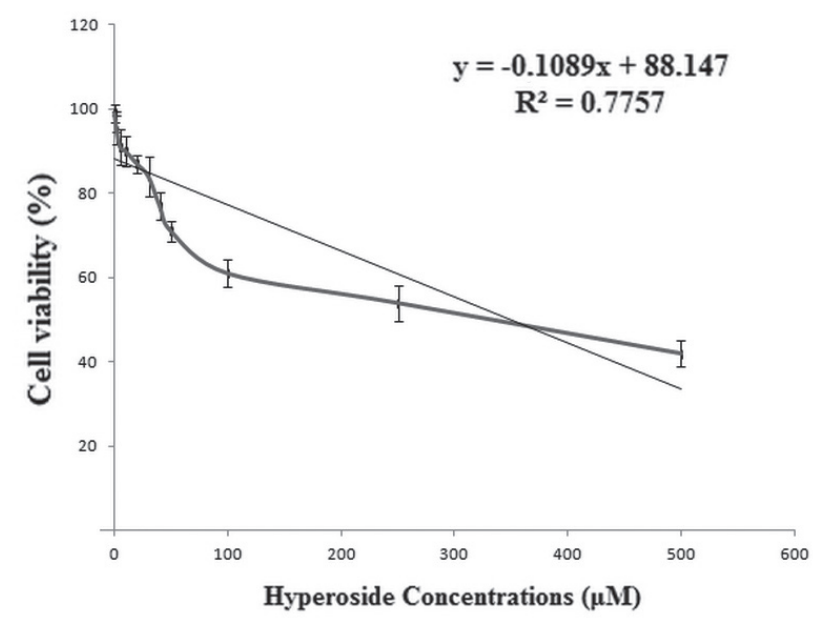

Figure 1. Effect of hyperoside on the cell viability of 3T3-L1 preadipocytes. Cells were plated in 96 well plate at density of $2 \times 10^{4}$ cells/well. The cells were pretreated with various concentrations of samples for $24 \mathrm{~h}$ and cytotoxicity was determined by MTT assay.
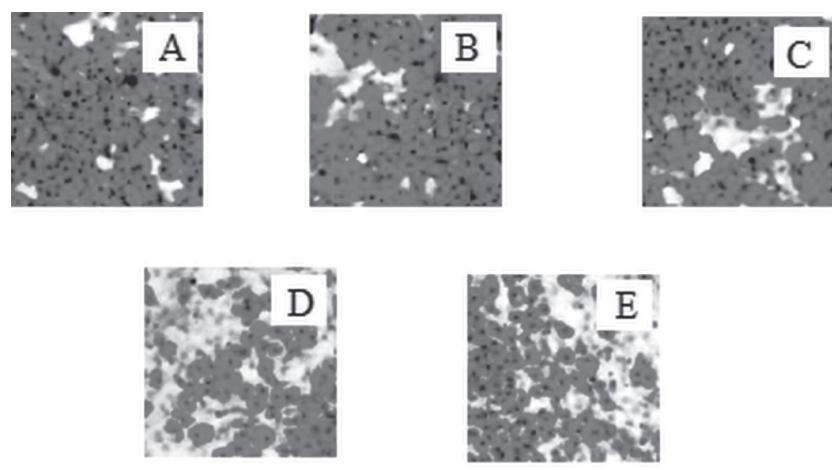

Figure 2. Effect of hyperoside on lipid accumulation during 3T3L1 adipocyte differentiation. A=Differentiate 3T3-L1 adipocytes (untreated adipocytes); B=Hyperoside at $1 \mu \mathrm{M}$ treated adipocytes; $\mathrm{C}=$ Hyperoside at $2 \mu \mathrm{M}$ treated adipocytes; $\mathrm{D}=$ Hyperoside at $5 \mu \mathrm{M}$ treated adipocytes; E=Hyperoside at $10 \mu \mathrm{M}$ treated adipocytes. The lipid droplets were observed at the magnification of 100x.

168 difluoride (PVDF) membrane (Millipore, Billerica, MA, USA), and treated with mouse anti- $\beta$-actin (1:5000), mouse anti-PPAR $(1: 1000)$, and mouse anti-SREBP1 (1:1000), (Santa-Cruz Biotech, CA, USA), and horseradish peroxidase (HRP)-conjugated secondary antibody (1:5000) for immunodetection. Relative protein expressions were measured by using ImageJ software programme (National Institutes of Health, Bethesda, MD, USA) (12).

\section{Statistical Analysis}

Statistical analyses were done in SPSS for Windows v15.0 package program. ANOVA was used to test the statistical differences between the groups for all parameters and Tukey multiple comparison tests were used for subgroup comparisons. Mean \pm standard deviation values were given as descriptive statistics and error bar graph was drawn for variables. Differences with a value of $\mathrm{p}<0.05$ were considered statistically significant.

\section{RESULTS}

\section{Investigation of the Cytotoxic Effect of Hyperoside on Preadipocytes}

In this study, we first examined the cytotoxic effect of hyperoside on preadipocytes in order to determine the therapeutic index. Cell death was assumed to be over $10 \%$ and hyperoside concentrations were cytotoxic. In the current study, hyperoside at concentrations of $1-500 \mu \mathrm{M}$ was applied to preadipocytes. According to the data we obtained, it was found that hyperoside at concentrations of 1-10 $\mu \mathrm{M}$ caused a maximum of $10 \%$ cytotoxicity, in other words, they did not show toxic effects. The effects of different concentrations of hyperoside on cell viability have been shown in Figure 1. In addition, the $\mathrm{LD}_{50}$ value of hyperoside for 3T3-L1 cells was found as $350.3 \mu \mathrm{M}$.

Investigation of the Effect of Hyperoside on Intracellular Triglyceride Accumulation

Oil Red $O$ staining was performed to investigate the effect of hyperoside on intracellular triglyceride accumulation in adipocytes. Hyperoside at concentrations of 5 and $10 \mu \mathrm{M}$ were found to suppress adipogenesis by significantly reducing intracellular triglyceride deposition (Fig. 2).

Considering all steps of adipogenesis, it was observed that all hyperoside concentrations except for $1 \mu \mathrm{M}$ decreased intracellular triglyceride content in a dose-dependent manner $(\mathrm{p}<0.05)$. The application of 5 
and $10 \mu \mathrm{M}$ hyperoside was the maximum concentration of intracellular glyceride content. $1 \mu \mathrm{M}$ hyperoside application inhibited intracellular triglyceride storage at the early stage of adipogenesis $(p<0.05)$, but it did not appear to be effective in the late period ( $p>0.05$ ). On the other hand, $10 \mu \mathrm{M}$ hyperoside administration was able to inhibit intracellular triglyceride storage only in the late period of adipogenesis $(p<0.05)$. When mature adipocytes were treated with hyperoside at a concentration of $1 \mu \mathrm{M}$, there was no statistically significant change in intracellular triglyceride content ( $p>0.05)$. On the other hand, hyperoside application to mature adipocytes at 2 and $5 \mu \mathrm{M}$ concentrations decreased intracellular triglyceride deposition, but this decrease was not statistically significant ( $p>0.05$ ). Hyperoside application at $10 \mu \mathrm{M}$ concentration was able to inhibit intracellular triglyceride content to the desired extent $(\mathrm{p}<0.05)$ (Fig. 3).

Investigation of the Effect of Hyperoside on the Transcription Factor Gene Expressions

In this study, hyperoside at concentrations of 5 and $10 \mu \mathrm{M}$, which had the most suppressing effect on adipogenesis, was used. It was observed that $5 \mu \mathrm{M}$ hyperoside suppressed the $C / E B P \beta$ and $P P A R \gamma$ expressions and $10 \mu \mathrm{M}$ hyperoside inhibited $P P A R \gamma$, SREBP- $1 c$ and $L P L$ expressions ( $\mathrm{p}<0.05)$ (Fig. 4).

Investigation of the Effect of Hyperoside on the Triacylglycerol Metabolism Gene Expressions

In mature adipocytes, gene expression studies were performed with hyperoside at a concentration of $10 \mu \mathrm{M}$, which had the most inhibitory effect

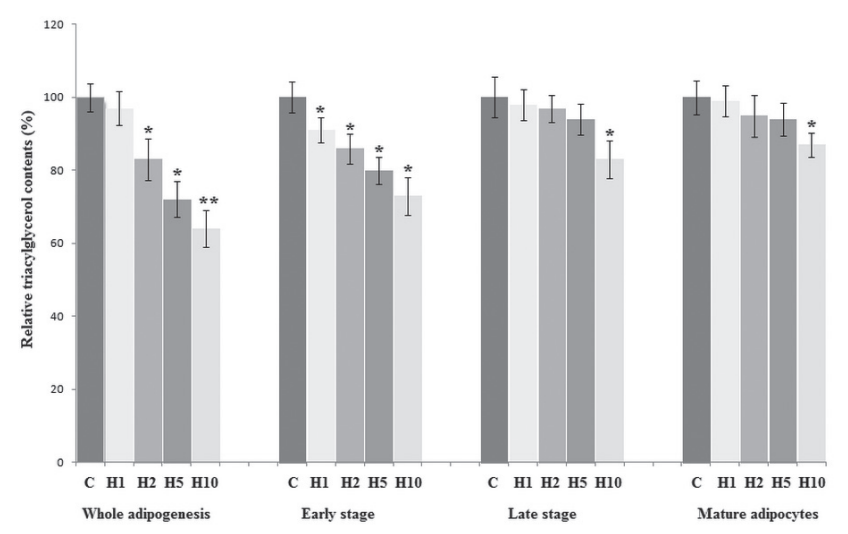

Figure 3. Relative triacylglycerol content (arbitrary units) after hyperoside treatment in maturing preadipocytes and mature adipocytes. $\mathrm{C}=$ control; $\mathrm{H} 1=1 \mu \mathrm{M}$ of hyperoside, $\mathrm{H} 2=2 \mu \mathrm{M}$ of hyperoside, $\mathrm{H} 5=5 \mu \mathrm{M}$ of hyperoside, and $\mathrm{H} 10=10 \mu \mathrm{M}$ of hyperoside. Values are means \pm SEM. Comparisons between each treatment and the controls were analyzed by Student's t-test. The asterisks represent differences versus the controls $\left({ }^{*} p<0.05 ;{ }^{*} p<0.001\right)$. on adipogenesis. HSL and FASN genes showed a statistically significant decrease $(\mathrm{p}<0.05)$, while no change was detected in the expression of $A C C, L P L$ and $A T G L$ genes $(\mathrm{p}>0.05)$. On the other hand, there was a statistically significant increase in expression of SIRT1 gene $(\mathrm{p}<0.05)$ (Fig. 5).

Investigation of the Effect of Hyperoside on the Transcription Factor Protein Expressions

The effects of 5 and $10 \mu \mathrm{M}$ hyperoside on protein expression of transcription factors of mature adipocytes were investigated. $5 \mu \mathrm{M}$ hyperoside did not alter the expression of PPAR 1 and PPAR 2 ( $\mathrm{p}>0.05)$, but caused an inhibition of SREBP1 expression $(\mathrm{p}<0.05) .10 \mu \mathrm{M}$ hyperoside significantly inhibited the expression of SREBP1 and PPAR $2(\mathrm{p}<0.05)$, but did not affect the expression of PPAR 1 ( $\mathrm{p}>0.05$ ) (Fig. 6).

Investigation of the Effect of Hyperoside on Adipokine Gene Expression

$10 \mu \mathrm{M}$ hyperoside treatment did not change the apelin, visfatin, leptin and adiponectin gene expressions on mature adipocytes (Fig. 7).

\section{DISCUSSION}

3T3-L1 cell line has been preferred in this study for investigation of the antiadipogenic potential of hyperoside. This cell line is a well-defined in vitro model for studying the adipogenesis mechanism. In the literature, both in vivo and in vitro anti-obesity activity of hyperoside has not been studied sufficiently $(13,14)$. In vitro models allow the investigation of the effects

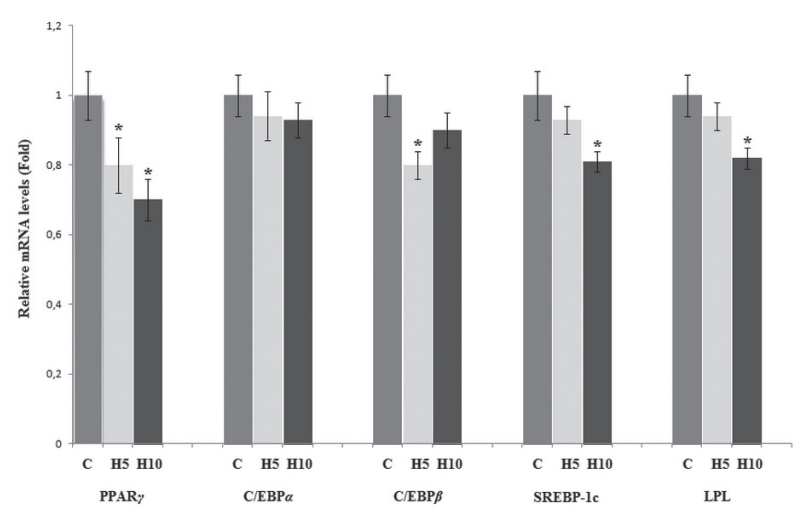

Figure 4. Effects of 5 and $10 \mu \mathrm{M}$ of hyperoside on gene expression of $C / E B P B, C / E B P \alpha, P P A R Y, S R E B P-1 C$, and $L P L$ in 3T3-L1 maturing preadipocytes treated from day 0 to day 8 . $C=$ control; $H 5=5 \mu \mathrm{M}$ of hyperoside, and $\mathrm{H} 10=10 \mu \mathrm{M}$ of hyperoside. Values are means \pm SEM. Comparisons between each treatment and the controls were analyzed by Student's t-test. The asterisks represent differences versus the controls $\left({ }^{*} p<0.05\right)$. 


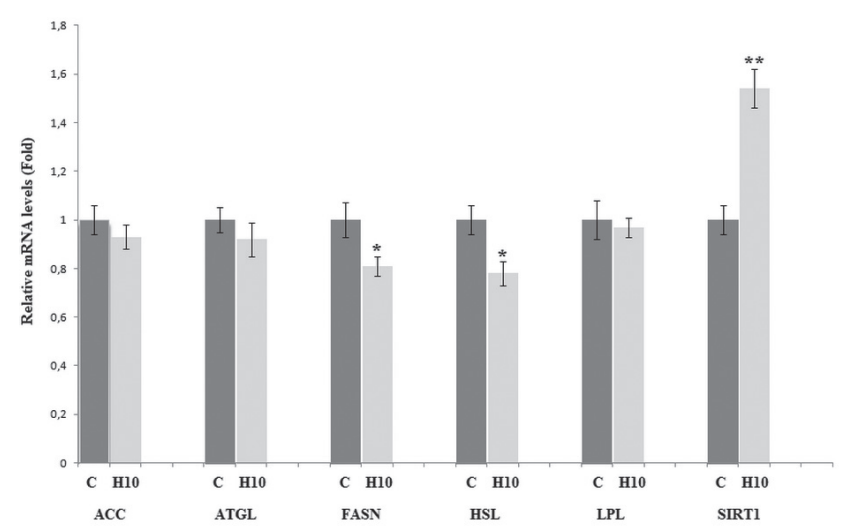

Figure 5. Effects of $10 \mu \mathrm{M}$ of hyperoside on the gene expression of HSL, FASN, ACC, LPL, ATGL, and SIRT1 in 3T3-L1 mature adipocytes treated for $24 \mathrm{~h}$. $\mathrm{C}=$ control; $\mathrm{H} 10=10 \mu \mathrm{M}$ of hyperoside. Values are means \pm SEM. Comparisons between each treatment and the controls were analyzed by Student's t-test. The asterisks represent differences versus the controls $\left({ }^{*} p<0.05 ;{ }^{*} p<0.001\right)$.

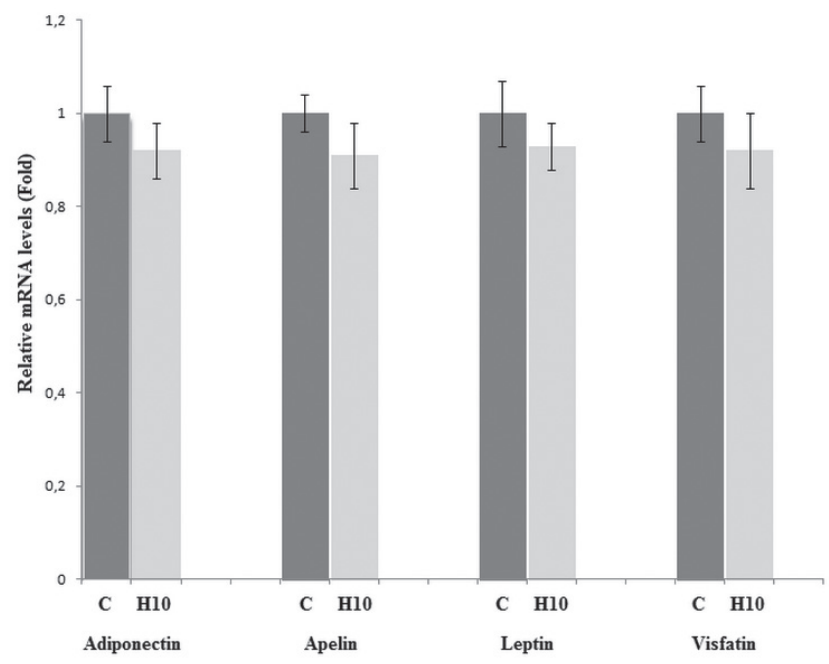

Figure 7. Effects of $10 \mu \mathrm{M}$ of hyperoside on the gene expression of adiponectin, leptin, visfatin, and apelin in 3T3-L1 mature adipocytes treated for $24 \mathrm{~h}$. $\mathrm{C}=$ control; $\mathrm{H} 10=10 \mu \mathrm{M}$ of hyperoside. Values are means \pm SEM. Comparisons between each treatment and the controls were analyzed by Student's t-test.

of the molecule on both preadipocytes and adipocytes at the molecular level. Therefore, we chose this experimental model in our study (12).

In the literature, there are several studies aiming to investigate the cytotoxic dose of hyperoside. Wang et al. 2016 (6) reported that high doses of hyperoside (0.5 to $2 \mathrm{mM})$ showed a highly cytoxic effect on human LX-2 hepatic stellate cells. In the current study, we investigated the cytotoxicity of hyperoside to determine the applicable treatment concentration range. According to the cytotoxicity test, $1-10 \mu \mathrm{M}$ of hyperoside concentrations showed maximum $10 \%$ cytotoxicity, so we decided to study with hyperoside in this dose range.

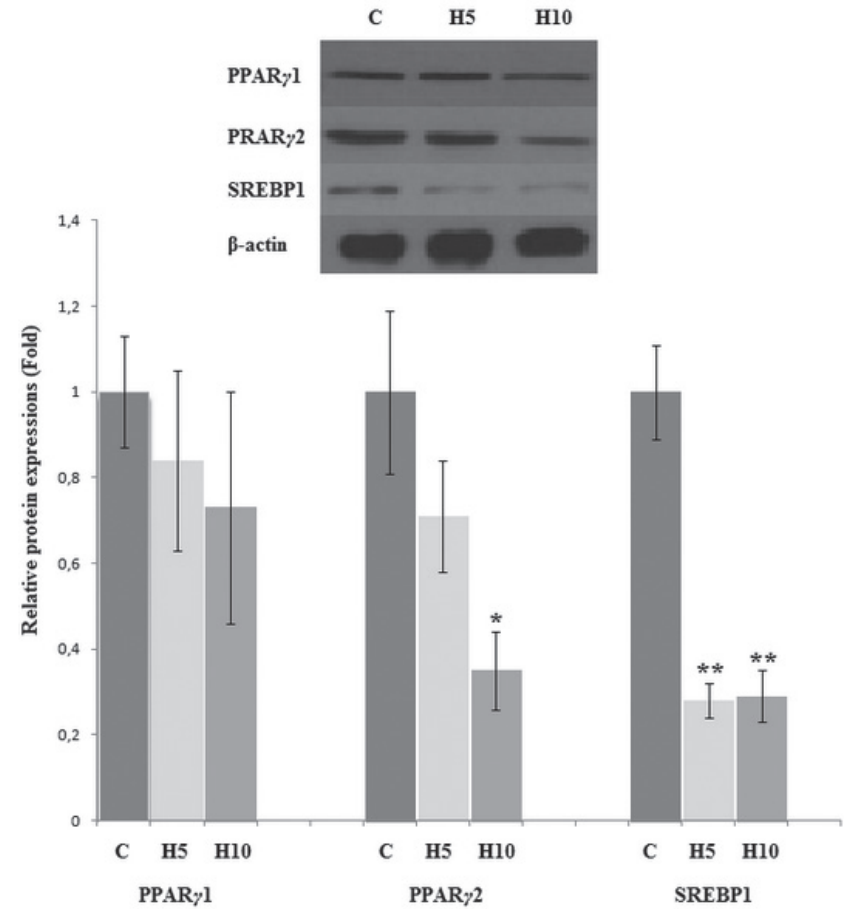

Figure 6. Effects of 5 and $10 \mu \mathrm{M}$ of hyperoside on protein expression of PPARY1, PPARY2, and SREBP-1C in 3T3-L1 maturing preadipocytes treated from day 0 to day 8 . $\mathrm{C}=$ control; $\mathrm{H} 5=5 \mu \mathrm{M}$ of hyperoside, and $\mathrm{H} 10=10 \mu \mathrm{M}$ of hyperoside. Values are means \pm SEM. Comparisons between each treatment and the controls were analyzed by Student's t-test. The asterisks represent differences versus the controls $\left({ }^{*} p<0.05 ; * * p<0.001\right)$.

Increased intracellular triglyceride stores play an important role in regulation of metabolic functions (11). In the current study, hyperoside administration caused differentiation of adipocytes but also increased intracellular triglycerides. On the other hand, application of hyperoside at a concentration of 5 and $10 \mu \mathrm{M}$ inhibited the accumulation of triglycerides in adipocyte cells, resulting in a slight change in cell morphology.

In our study, it was observed that all hyperoside concentrations except $1 \mu \mathrm{M}$ decreased the intracellular triglyceride content of preadipocytes depending on the dose. In mature adipocytes, hypersite at only $10 \mu \mathrm{M}$ concentration decreased intracellular triglyceride content. Park et al. (15) obtained similar results with $25 \mu \mathrm{M}$ of quercetin concentration. According to these data, it is possible to say that hyperoside inhibits adipogenesis even at lower doses than quercetin. To our best knowledge, there has been no study that investigated the effect of hyperoside on adipogenesis in vitro. However, there have been many studies investigating the effect of quercetin that has a similar structure to hyperoside on 3T3-L1 preadipocytes $(16,17)$. Ahn et al. (16) applied 10,50 and $100 \mu \mathrm{M}$ quercetin to preadipocytes and 
found that all three concentrations lower intracellular triglyceride content. Yang et al. (17) applied 12.5 and $25 \mu \mathrm{M}$ quercetin to preadipocytes, but they observed that only $25 \mu \mathrm{M}$ quercetin concentration could inhibit intracellular triglyceride storage.

Hyperoside administration at a concentration of $5 \mu \mathrm{M}$ significantly reduced the $C / E B P \beta$ and $P P A R \gamma$ gene expression, but did not alter PPAR $\gamma 1$ and $P P A R \gamma 2$ protein levels. In addition, a decrease in protein expression of SREBPI was observed. $C / E B P \beta$ and $S R E B P-1 C$ are among the transcription factors involved in the first stage of adipogenesis. These data show that adipogenesis stops at early stage. This was evidenced by the decrease in the amount of intracellular triglycerides observed in the early stage of differentiation.

In the current study, PPAR $\gamma$ and SREBP-1c gene and protein expression and $L P L$ gene expression of adipocytes treated with $10 \mu \mathrm{M}$ hyperoside decreased compared to control. Ahn et al. (16) and Yang et al. (17) found a decrease in PPAR $\gamma$ and SREBP-1c expressions of adipocytes treated with 10 and $25 \mu \mathrm{M}$ quercetin in two different studies. Approximately 5-6 days after adipocyte cells are confluent, SREBP$1 c$ expression in adipocytes reaches the maximum level (18). Therefore, as in PPAR, SREBP-1c is an important transcription factor involved in the late stages of adipogenesis (19). Treatment of adipocytes with hyperoside at a concentration of $10 \mu \mathrm{M}$, leading to a decrease in PPAR $\gamma$ and $S R E B P-1 c$ expressions and intracellular triglyceride content, shows the effect of hyperoside at this concentration on the terminal stage of adipogenesis.

Expression of genes involved in triglyceride metabolism was measured after 24 hours of administration of different concentrations of hyperoside to mature adipocytes. Hyperoside administration decreased HSL and FASN expression but it did not change ATGL expression. This may be due to the lipid lowering effect of this molecule by suppressing de novo lipogenesis. Chylomicrons and VLDLs are circulated throughout the bloodstream and upon arrival at adipose tissue, the triglycerides contained in these lipoproteins are cleaved by $L P L$ into glycerol and free fatty acids. Once inside adipocytes, free fatty acids are subsequently esterfied to new triglycerides (20). In our study, hyperoside administration did not cause significant changes in $L P L$ gene expression in mature adipocytes.

It is known that many positive effects of polyphenolic compounds are achieved by deacetylase
SIRT1 (21). In our study, it was observed that the application of $10 \mu \mathrm{M}$ concentration of hyperoside to mature adipocytes caused an increase in SIRT1 expression. When deacetylation of $S R E B P-1 c$ is induced by $S I R T 1$, transcriptional activation of $S R E B P-1 c$ can inhibit and thus result in a decrease in the expression of lipogenic proteins (22). Therefore, we think that the increase in SIRT1 expression in mature adipocytes may be due to the decrease in FASN expression.

Flavonoids have been reported to reduce insulin resistance by reducing the expression of adipokines (23-25). It has been found that quercetin reduces visfatin expression in adipocytes and increases adiponectin expression (26). To our knowledge, the effects of hyperoside administration to adipocytes on the expression of adipokines have not been studied. In the current study, we think that our hyperoside concentrations do not cause any change in adipokine gene expression and may be due to insufficiency of hyperosidine application concentrations on glycemic control mediators.

In conclusion, it was observed that noncytotoxic concentrations of hyperoside decreased intracellular triglyceride accumulation and suppressed adipogenesis by inhibiting $P P A R \gamma, C / E B P \beta$ and $S R E B P-1 c$ expression. However, it was found that low concentrations of hyperoside $(5 \mu \mathrm{M})$ may be sufficient to suppress adipogenesis, but high concentrations of hyperoside $(10 \mu \mathrm{M})$ are needed to prevent intracellular triglyceride accumulation in mature adipocytes. All these results indicate that hyperoside can be used in the prevention and treatment of obesity.

\section{Conflict of interest}

The author declares that he has no conflict of interest.

\section{Acknowledgement}

This research was financially supported in part by the Office of Scientific Research Projects of Yuzuncu Yil University under Grant number (TAP-2018-6956).

\section{References}

1. Engin A. The definition and prevalence of obesity and metabolic syndrome. Adv Exp Med Biol 2017; 960:1-17.

2. Ziylan YZ, Baltaci AK, Mogulkoc R. Leptin transport in the central nervous system. Cell Biochem Funct 2009; 27(2):63-70.

3. Mokdad AH, Ford ES, Bowman BA, Dietz WH, Vinicor F, Bales VS, Marks JS. Prevalence of obesity, diabetes, and obesity-related health risk factors, 2001. JAMA 2003; 289(1):76-79.

4. Wei WY, Zhang N, Tang QZ. The potential role of PPAR $\gamma$ in obesity-induced adipose tissue inflammation. Int J Cardiol 2018; 266:220. 
5. D'Andrea G. Quercetin: A flavonol with multifaceted therapeutic applications? Fitoterapia 2015; 106:256-271.

6. Baltaci SB, Mogulkoc R, Baltaci AK. Resveratrol and exercise. Biomed Rep 2016; 5(5):525-530.

7. Wang L, Yue Z, Guo M, Fang L, Bai L, Li X, Tao Y, Wang S, Liu Q, Zhi D, Zhao H. Dietary flavonoid hyperoside induces apoptosis of activated human LX-2 hepatic stellate cell by suppressing canonical NF- $\kappa B$ signaling. Biomed Res Int 2016; 2016:1068528.

8. Lasa A, Churruca I, Eseberri I, Andrés-Lacueva C, Portillo MP. Delipidating effect of resveratrol metabolites in 3T3-L1 adipocytes. Mol Nutr Food Res 2012; 56(10):1559-1568.

9. Eseberri I, Lasa A, Churruca I, Portillo MP. Resveratrol metabolites modify adipokine expression and secretion in 3T3-L1 pre-adipocytes and mature adipocytes. PLoS One 2013; 8(5):e63918.

10. Kang JW, Nam D, Kim KH, Huh JE, Lee JD. Effect of gambisan on the inhibition of adipogenesis in 3T3-L1 adipocytes. Evid Based Complement Alternat Med 2013; 2013:789067.

11. Li Y, Rong Y, Bao L, Nie B, Ren G, Zheng C, Amin R, Arnold RD, Jeganathan RB, Huggins KW. Suppression of adipocyte differentiation and lipid accumulation by stearidonic acid (SDA) in 3T3-L1 cells. Lipids Health Dis 2017; 16(1):181.

12. Fromm-Dornieden C, von der Heyde S, Lytovchenko O, SalinasRiester G, Brenig B, Beissbarth T, Baumgartner BG. Novel polysome messages and changes in translational activity appear after induction of adipogenesis in 3T3-L1 cells. BMC Mol Biol 2012; 13:9.

13. Li S, Zhang Z, Cain A, Wang B, Long M, Taylor J. Antifungal activity of camptothecin, trifolin, and hyperoside isolated from Camptotheca acuminata. J Agric Food Chem 2005; 53(1):32-37.

14. Zhang Y, Wang M, Dong H, Yu X, Zhang J. Anti-hypoglycemic and hepatocyte-protective effects of hyperoside from Zanthoxylum bungeanum leaves in mice with high-carbohydrate/high-fat diet and alloxan-induced diabetes. Int J Mol Med 2018; 41(1):77-86.

15. Park HJ, Yang JY, Ambati S, Della-Fera MA, Hausman DB Rayalam S, Baile CA. Combined effects of genistein, quercetin, and resveratrol in human and 3T3-L1 adipocytes. J Med Food 2008; 11(4):773-783.
16. Ahn J, Lee H, Kim S, Park J, Ha T. The anti-obesity effect of quercetin is mediated by the AMPK and MAPK signaling pathways. Biochem Biophys Res Commun 2008; 373(4):545-549.

17. Yang JY, Della-Fera MA, Rayalam S, Ambati S, Hartzell DL, Park HJ, Baile CA. Enhanced inhibition of adipogenesis and induction of apoptosis in 3T3-L1 adipocytes with combinations of resveratrol and quercetin. Life Sci 2008; 82(19-20):1032-1039.

18. Dowell P, Flexner C, Kwiterovich PO, Lane MD. Suppression of preadipocyte differentiation and promotion of adipocyte death by HIV protease inhibitors. J Biol Chem 2000; 275(52):41325-41332.

19. Rangwala SM, Lazar MA. Transcriptional control of adipogenesis. Annu Rev Nutr 2000; 20:535-559.

20. Wang H, Eckel RH. Lipoprotein lipase: from gene to obesity. Am J Physiol Endocrinol Metab 2009; 297(2):E271-288.

21. Chung S, Yao H, Caito S, Hwang JW, Arunachalam G, Rahman I. Regulation of SIRT1 in cellular functions: role of polyphenols. Arch Biochem Biophys 2010; 501(1):79-90.

22. Ponugoti B, Kim DH, Xiao Z, Smith Z, Miao J, Zang M, Wu SY, Chiang CM, Veenstra TD, Kemper JK. SIRT1 deacetylates and inhibits $S R E B P-1 c$ activity in regulation of hepatic lipid metabolism. J Biol Chem 2010; 285(44):33959-33970.

23. Ahmadpour F, Nourbakhsh M, Razzaghy-Azar M, Khaghani S, Alipoor B, Abdolvahabi Z, Zangoei M. The association of plasma levels of mir-34A and mir-149 with obesity and insulin resistance in obese children and adolescents. Acta Endocrinol (Buchar) 2018; 14(2):149-154.

24. Derdemezis CS, Kiortsis DN, Tsimihodimos V, Petraki MP, Vezyraki P, Elisaf MS, Tselepis AD. Effect of plant polyphenols on adipokine secretion from human SGBS adipocytes. Biochem Res Int 2011; 2011:285618.

25. Naraoka Y, Yamaguchi T, Hu A, Akimoto K, Kobayashi H. Short chain fatty acids upregulate adipokine production in type 2 diabetes derived human adipocytes. Acta Endocrinologica-Bucharest 2018; 14(3):287-293.

26. Wein S, Behm N, Petersen RK, Kristiansen K, Wolffram S. Quercetin enhances adiponectin secretion by a PPAR-gamma independent mechanism. Eur J Pharm Sci 2010; 41(1):16-22. 